\title{
Ultimate Boundedness Results for Noise-Corrupted Quaternion Output Feedback Attitude Tracking Controllers
}

\author{
Sungpil Yang* \\ University of Texas at Austin, Austin, Texas, 78712 \\ Frédéric Mazenc ${ }^{\dagger}$
}

Equipe Projet Inria Dynamical Interconnected Systems in Complex Environments, 91192, Gif-sur-Yvette, Franc

and

Maruthi R. Akella

University of Texas at Austin, Austin, Texas, 78712

\section{Introduction}

The importance of the rigid body attitude control problem is well established with a wide range of applications from robot manipulators to satellites and continues to receive significant attention in recent literature. In spite of the technical difficulties of this problem that stem from mathematical description of the attitude and the nonlinearity of the dynamics, this subject has been extensively studied and numerous solutions proposed over the past two decades [1]: Three-parameter representations like Euler angles, Rodrigues Parameters (RPs) and Modified Rodrigues Parameters (MRPs) suffer from the kinematic singularity, and singularity-free quaternions are limited by the nonlinear unit norm constraint. In Euler's rotational dynamics, the formulation is more complex due to the

${ }^{*}$ Ph.D. Candidate, Department of Aerospace Engineering and Engineering Mechanics, 1 University Station; yang. sungpil@utexas. edu. Student Member AIAA

${ }^{\dagger}$ Chargé de Recherche, EPI DISCO Inria, Laboratoire des Signaux et Systèmes (L2S, UMR CNRS 8506), CNRS, CentraleSupélec, Université Paris-Sud, 3 rue Joliot Curie; frederic mazenc@l2s . centralesupelec. fr.

${ }^{*}$ Professor, Department of Aerospace Engineering and Engineering Mechanics, 1 University Station. Myron L. Begeman Fellow in Engineering; makella@mail.utexas. edu. Associate Fellow AIAA 
Coriolis term.

Both stabilizing and tracking solutions, using various attitude representations, are now well understood - especially for full-state feedback control systems [1-5]. However, full-state feedback is not always possible due to cost limitations or design constraints. In the cases where rate-gyro measurements do not directly appear in the control law or gyro failure occurs during the mission, passivity-based approaches have been suggested for alternate solutions. High-gain observers or numerical differentiation schemes are another options, but the closed-loop system may be subject to peaking phenomenon or noise inversely proportional to a sampling time.

Attitude stabilization without using the rate state or its estimate was originally proposed by Lizarralde and Wen via the passivity framework [6]. A stable filter driven by attitude signals is considered to achieve a passive system interconnection, and both input and output of the filter are employed to design a velocity-free control law. Many further extensions including different parameterizations and generalization to tracking control have appeared in the literature [7-12].

Since passivity-based velocity-free controllers are model independent for the set-point regulation problem, robustness properties to inertia parameter uncertainties automatically follow. For the tracking case, however, the controller is dependent on the inertia matrix so it can be subject to model mismatch. In the literature, output feedback adaptive attitude tracking control is studied in [13], which is automatically robust to the inertia uncertainty. In addition to system identification error, these controllers are, in general, subject to other types of uncertainty. In reality, the body orientation cannot be perfectly measured and external disturbance torques always exist.

The attitude states are determined directly from line-of-sight measurements or are estimated using filters [14]. Accelerometers, magnetometers and star sensors can provide vector measurements that determine the body orientation through deterministic methods like TRIAD, QUEST or FOAM in a way that measurement errors are minimized [15-17]. Sometimes rate-gyros are used to filter out measurement noise [14]. Recently, the velocity-free stabilization problem using vector measurements instead of converting them to the attitude variables was addressed, but measurement errors are excluded in their analyses $[18,19]$.

Environmental disturbances within a feedback loop are another source of uncertainty. Solar 
radiation pressure, atmospheric drag, earth-oblateness or gravity gradient effects can all generate non-negligible external torques that perturb the body orientation. To deal with this robustness question, the attitude control problem is typically formulated by including an additive bounded disturbance term in the Euler rotational dynamics. In the literature, on one hand, several disturbancerejection controllers are proposed using modified filters: [9] designed a control algorithm where integral terms appear both in the controller and the filter while [11] proposed a non-smooth control achieving semi-global stability in the presence of disturbances. On the other hand, in [20,21], full-state and observer-based output feedback controllers with exponential gains were proposed establishing the closed-loop systems are Uniformly Practically Asymptotically Stable (UPAS) when disturbance torques exist (roughly speaking, UPAS means asymptotic stability of a ball whose radius becomes smaller by tuning controller gains [20-22]). More recently, [23] proposed a velocityfree output feedback tracking controller that is robust to bounded external disturbance torques.

When full states are available, [24] proposed nonlinear tracking controllers with various attitude representations for a spacecraft carrying an unknown large object which significantly influences inertia parameters. In the presence of modeling uncertainties, measurement errors and disturbance torques, the controllers guarantee global exponential convergence of tracking errors with finitegain $\mathcal{L}_{p}$ stability. In this paper, we propose a passivity-based output feedback tracking controller with the quaternion kinematics while considering measurement errors, disturbance torques and parameter uncertainties. The proposed controller achieves asymptotic stability of the tracking errors in the ideal case and guarantees ultimate boundedness of the closed-loop trajectories through proper gains that can be selected from stability analysis.

Since the problem is formulated with the unit-quaternion representation, we can avoid geometric singularity normally caused by minimal three-dimensional parameterizations. Compared to the global representation of attitude kinematics described by direction cosine matrices, it does not require high-dimensional formulation, and thus, higher-order numerical renormalization to satisfy the orthogonality constraint. Unlike the control laws with the quaternion kinematics in [24] and ones in $[13,23]$, the proposed controller does not include control singularity which occurs when the scalar part of the error quaternion becomes zero. Although those controllers are nonsingular if 
they are initially, quaternion tracking errors in the presence of measurement uncertainties around the singular point may lead to undesirable behavior. Specifically, to achieve disturbance rejection, one of the gains of the controller in [23] becomes arbitrarily large even for arbitrarily small disturbances if the initial conditions are close to the singular point; therefore, appropriate reference design must precede the gain selection to avoid any potential singularity issues.

This work builds on the preliminary results from $[25,26]$ by generalizing to the tracking case and some further analysis of the filter-free case. Extending the strictification technique (see Refs. [27-29] and the references therein for details), a partially strict Lyapunov function is constructed toward establishment of stability and ultimate boundedness properties for the closed-loop system. With known upper bounds of the magnitude of measurement errors, disturbance torques, and parameter uncertainties, a feasible range for the feedback gains is derived in terms of bounds on the initial conditions in such a way to ensure asymptotic convergence of all closed-loop signals to within a residual set. Since the system is not input-to-state stable, boundedness properties do not come for free when all the uncertainties and disturbance torques are considered. In spite of the nonlinear structure of the kinematics and dynamics of the problem, however, the closed-loop system is rigorously analyzed through the standard Lyapunov analysis methods. This is achieved owing to the fact that the strictified Lyapunov function allows us to deal with this nontrivial problem in a standard way. As the passivity-based controller is not new for the attitude control problem, the key contribution of this paper would be a theoretical proof how ideal-case design is robust to uncertainties through Lyapunov stability analysis.

The paper is organized as follows. Euler's rotational dynamics with the quaternion kinematics are described in Section II and the problem is formulated in Section III. Next, a velocity-free tracking controller is proposed and then the closed-loop system is analyzed in the presence of the uncertainties in Section IV. Numerical results for stabilization and tracking cases are provided in Section V. Finally, Section VI presents some concluding remarks. 


\section{Equations of Motion}

\section{A. Quaternion}

Let $\mathcal{F}_{I}$ be the inertial frame and $\mathcal{F}_{B}$ be a body-fixed frame. Let $\mathcal{F}_{R}$ be a reference frame with which the body-fixed frame is required to be aligned by a controller. The body orientation is assumed to be measured in such a way that it is different from the true orientation due to measurement errors or estimation errors. So we consider an estimated body-fixed frame $\hat{\mathcal{F}}_{B}$. Let $q, \hat{q}$ and $q_{r}$ be the quaternions representing the orientations of $\mathcal{F}_{B}, \hat{\mathcal{F}}_{B}$ and $\mathcal{F}_{R}$ with respect to the inertial frame $\mathcal{F}_{I}$. The rotations from $\mathcal{F}_{R}$ to $\mathcal{F}_{B}$ and $\hat{\mathcal{F}}_{B}$ are represented by the quaternions $\delta q$ and $\delta \hat{q}$. Also the error between true and estimated body orientations is denoted as $\delta \tilde{q}$. The aforementioned quaternions and their corresponding frame rotations are described in Fig.1.
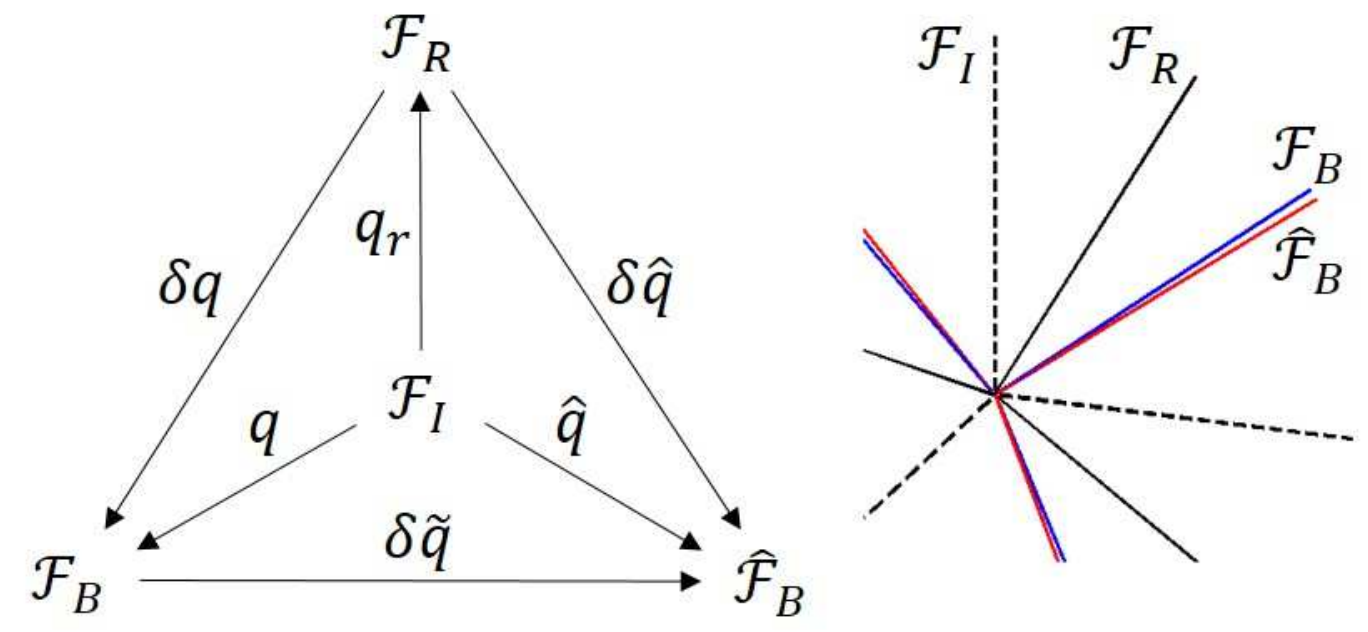

Figure 1. Frames and corresponding quaternions

Quaternions are converted to rotation matrices. Let $C: \mathbb{Q} \rightarrow S O(3)$ be a map defined as [30]

$$
C(q)=I_{3}-2 q_{0} q_{v}^{*}+2 q_{v}^{*} q_{v}^{*}
$$

where the domain $\mathbb{Q}=\left\{q=\left(q_{0}, q_{v}\right) \in \mathbb{R}^{4} \mid q_{0} \in \mathbb{R}, q_{v} \in \mathbb{R}^{3}, q^{T} q=1\right\}$ is the set of all quaternions and the codomain $S O(3)=\left\{R \in \mathbb{R}^{3 \times 3} \mid R^{T} R=R R^{T}=I_{3}, \operatorname{det}(R)=1\right\}$ is the three dimensional rotation group, $I_{3}$ is the three-dimensional identity matrix and the superscript ${ }^{*}$ is the skew-symmetric matrix operator such that $v^{*} w=v \times w$ for any $v, w \in \mathbb{R}^{3}$. Note that since the quaternion parameter- 
ization is defined over non-Euclidean space, it is free from the mathematical singularity that arises from all minimal three-parameter representations.

Since successive rotations can be represented as one rotation, the rotation from $\mathcal{F}_{I}$ to $\mathcal{F}_{B}$ is represented as

$$
C(q)=C(\delta q) C\left(q_{r}\right)
$$

Equivalently, the quaternion $q$ is expressed as

$$
q=G\left(q_{r}\right) \delta q
$$

where $G: \mathbb{Q} \rightarrow \mathbb{R}^{4 \times 4}$ is defined as

$$
G(q)=\left[\begin{array}{cc}
q_{0} & -q_{v}^{T} \\
q_{v} & q_{v}^{*}+q_{0} I_{3}
\end{array}\right]
$$

Note that one can easily derive $G$ is an orthogonal matrix from the definition. The true quaternion tracking error is then given by

$$
\delta q=G^{T}\left(q_{r}\right) q
$$

which corresponds to

$$
C(\delta q)=C(q) C^{T}\left(q_{r}\right)
$$

The role of tracking controllers is to make $C(\delta q)$ converge to $I_{3}$. In quaternion representation, that means $\delta q_{v}$ becomes zero whatever its scalar part is.

\section{B. Kinematics}

The kinematic equation for the body attitude described in terms of the quaternion $q$ is given by

$$
\dot{q}(t)=\frac{1}{2} E(q(t)) \omega(t),
$$


where $\omega$ is the angular rate of the body with respect to the body-fixed frame $\mathcal{F}_{B}$ and the map $E: \mathbb{Q} \rightarrow \mathbb{R}^{4 \times 3}$ is given by

$$
E(q)=\left[\begin{array}{c}
-q_{v}^{T} \\
F(q)
\end{array}\right]
$$

with

$$
F(q)=q_{v}^{*}+q_{0} I_{3}
$$

Let the reference angular rate in the frame $\mathcal{F}_{R}$ be denoted as $\omega_{r}$ which is assumed to be smooth and bounded so that we have

$$
\begin{aligned}
& \sup _{t \geq 0}\left\|\omega_{r}(t)\right\|_{2}=v_{\omega}, \\
& \sup _{t \geq 0}\left\|\dot{\omega}_{r}(t)\right\|_{2}=v_{\alpha},
\end{aligned}
$$

for some finite positive numbers $v_{\omega}$ and $v_{\alpha}$. It is also assumed that the reference states are governed by

$$
\dot{q}_{r}(t)=\frac{1}{2} E\left(q_{r}(t)\right) \omega_{r}(t) .
$$

Let $\delta \omega$ be the difference between the actual and desired rate in the body-fixed frame $\mathcal{F}_{B}$. Then the rate error is defined as

$$
\delta \omega(t)=\omega(t)-\omega_{r}^{B}(t)
$$

where

$$
\omega_{r}^{B}(t)=C(\delta q(t)) \omega_{r}(t)
$$

By differentiating Eq. (6) with respect to time, we obtain [31]

$$
\delta \dot{q}(t)=\frac{1}{2} E(\delta q(t)) \delta \omega(t)
$$




\section{Dynamics}

The mathematical model for the fully-actuated rigid-body rotational motion is described by Euler's equations given by

$$
J \dot{\omega}(t)=-\omega^{*}(t) J \omega(t)+u(t)
$$

where $J=J^{T} \in \mathbb{R}^{3 \times 3}$ is the moment-of-inertia matrix and $u(t) \in \mathbb{R}^{3}$ is the input torque. Note that, when we let $J_{M}$ and $J_{m}$ be the largest and the smallest eigenvalues of $J$ respectively, $J$ satisfies

$$
J_{m}\|v\|_{2}^{2} \leq v^{T} J v \leq J_{M}\|v\|_{2}^{2}
$$

for all $v \in \mathbb{R}^{3}$.

From Eq. (13), the error dynamics are derived as

$$
J \delta \dot{\omega}(t)=-\omega^{*}(t) J \omega(t)+u(t)+J \delta \omega^{*}(t) \omega_{r}^{B}(t)-J C(\delta q(t)) \dot{\omega}_{r}(t)
$$

Alternatively, from the identity

$$
(J \delta \omega)^{*}+\left(J \delta \omega^{*}\right)+\left(\delta \omega^{*} J\right)=\operatorname{tr}(J) \delta \omega^{*},
$$

where $\operatorname{tr}(J)$ is the trace of $J$, we have

$$
J \delta \dot{\omega}(t)=-\delta \omega^{*}(t)\left(J \delta \omega(t)+\bar{J} \omega_{r}^{B}(t)\right)+u(t)-\eta(t)
$$

with

$$
\bar{J}=2 J-\operatorname{tr}(J) I_{3}
$$

and

$$
\eta(t)=\omega_{r}^{B *}(t) J \omega_{r}^{B}(t)+J C(\delta q(t)) \dot{\omega}_{r}(t)
$$




\section{Problem Description}

We assume that the system is equipped with only attitude sensors so that full-state feedback control is not feasible. In spite of the lack of gyro sensors, it is well known that the system can be stabilized utilizing passivity-based control strategies for which a low-pass filter driven by the quaternion signal is added to the original dynamics $[6,7]$. Now we consider, in the presence of uncertainties, the stabilization of the system

$$
\begin{aligned}
\delta \dot{q}(t) & =\frac{1}{2} E(\delta q(t)) \delta \omega(t) \\
J \delta \dot{\omega}(t) & =-\delta \omega^{*}(t)\left(J \delta \omega(t)+\bar{J} \omega_{r}^{B}(t)\right)+u(t)-\eta(t)+d(t) \\
\dot{\sigma}(t) & =-\sigma(t)+\delta \hat{q}(t),
\end{aligned}
$$

where $\delta \hat{q}$ is the estimated quaternion tracking error and $d(t)$ is a bounded disturbance: there is a constant $\mu_{d}>0$ such that, for all $t \geq 0$, the inequality

$$
\|d(t)\|_{2} \leq \mu_{d}
$$

is satisfied. Also, it is assumed that the exact value of $J$ is not available so its estimate $\hat{J}$ is used for the control. Our objective is to show a passivity based PD-type control law stabilizing the system in the ideal situations guarantees the boundedness of the closed-loop trajectories in the presence of measurement errors, disturbance torques and inertia parameter uncertainties.

From Fig. 1, the attitude estimate error $\delta \tilde{q} \in \mathbb{Q}$ is obtained as

$$
\delta \tilde{q}(t)=G^{T}(\delta q(t)) \delta \hat{q}(t)
$$

We assume that it is governed by the following dynamics:

$$
\delta \dot{\tilde{q}}(t)=\frac{1}{2} E(\delta \tilde{q}(t)) \tilde{\omega}(t)
$$

where the forcing term $\tilde{\omega}$ is a random variable [32]. Observe that this assumption allows the 
quaternion estimate $\hat{q}(t)$ to remain in the set $\mathbb{Q}$ for all $t \geq 0$. We also assume, for the analysis, that $\tilde{\omega}$ is bounded by a known quantity and restricts the magnitude of $\delta \tilde{q}_{v}$ within a small number:

$$
\begin{gathered}
\left\|\delta \tilde{q}_{v}(t)\right\|_{2} \leq \mu_{v} \ll 1, \\
\|\tilde{\omega}(t)\|_{2} \leq 2 \mu_{\omega} \ll 1
\end{gathered}
$$

for all $t \geq 0$. Typically $\mu_{v}$ and $\mu_{\omega}$ are very small quantities which are available based on the sensor characteristics. Due to the unit-norm constraint of the error quaternion $\delta \tilde{q}(t)$, its scalar part $\delta \tilde{q}_{0}(t)$ is bounded by

$$
\mu_{0} \leq \delta \tilde{q}_{0}(t) \leq 1
$$

where $\mu_{0}=\sqrt{1-\mu_{v}^{2}}$ with the assumption $\delta \tilde{q}_{0}(0)>0$. In other words, $\delta \tilde{q}$ is very close to the identity quaternion $(1,0,0,0)$.

Throughout the paper, $x=(\delta q, \delta \omega, \dot{\sigma}) \in \mathbb{Q} \times \mathbb{R}^{7}$ represents the entire states and $\bar{x}=\left(\delta q_{v}, \delta \omega, \dot{\sigma}\right) \in$ $\mathbb{R}^{10}$ the states of interest to be stabilized. We also introduce the notations $z=\left(\left\|\delta q_{v}\right\|_{2},\|\delta \omega\|_{2},\|\dot{\sigma}\|_{2}\right) \in$ $\mathbb{R}^{3}$ and $\bar{z}=\left(\|\delta \omega\|_{2},\|\dot{\sigma}\|_{2}\right) \in \mathbb{R}^{2}$ for later use. Arguments of functions are often omitted for notational simplicity whenever no confusion arises within a context.

\section{Main Result}

In this section, boundedness of the solution of Eq. (23) is established through a partially strictified Lyapunov function in the presence of quaternion estimate errors, disturbance torques and parameter uncertainties.

Let us consider the attitude dynamics (23) with $\delta \hat{q}(t)=\delta q(t)$ and $d(t)=0$, and assume that the initial conditions are such that $\|\delta q(0)\|_{2}=1, \delta \omega(0) \in \mathbb{R}^{3}$ and $\dot{\sigma}(0)=0$. Then, by analyzing

$$
V_{0}(t)=\mathfrak{a}\left[\delta q_{v}^{T}(t) \delta q_{v}(t)+\left(\delta q_{0}(t)-1\right)^{2}\right]+\frac{1}{2} \delta \omega^{T}(t) J \delta \omega(t)+\frac{\mathfrak{b}}{2} \dot{\sigma}^{T}(t) \dot{\sigma}(t)
$$

where $\mathfrak{a}$ and $\mathfrak{b}$ are positive constants, one can show that the control

$$
u(t)=-\mathfrak{a} \delta q_{v}(t)-\frac{\mathfrak{b}}{2} E^{T}(\delta q(t)) \dot{\sigma}(t)+\eta(t)
$$


stabilizes the system (23) [25].

When the uncertainties are considered, we employ $\delta \hat{q}(t)$ and $\hat{J}$ instead of $\delta q(t)$ and $J$ respectively for the controller and the filter. Then the proposed control law is rewritten as

$$
\begin{aligned}
u(t) & =-\mathfrak{a} \delta \hat{q}_{v}(t)-\frac{\mathfrak{b}}{2} E^{T}(\delta \hat{q}(t)) \dot{\sigma}(t)+\hat{\eta}(t) \\
& =-\mathfrak{a}\left[\delta \tilde{q}_{0}(t) \delta q_{v}(t)+F(\delta q(t)) \delta \tilde{q}_{v}(t)\right]+\frac{\mathfrak{b}}{2} E^{T}(\dot{\sigma}(t)) G(\delta q(t)) \delta \tilde{q}(t)+\hat{\eta}(t),
\end{aligned}
$$

where $\hat{\eta}(t)=\left[C(\delta \hat{q}(t)) \omega_{r}(t)\right]^{*} \hat{J} C(\delta \hat{q}(t)) \omega_{r}(t)+\hat{J} C(\delta \hat{q}(t)) \dot{\omega}_{r}(t)$. The filter becomes

$$
\begin{aligned}
\dot{\sigma}(t) & =-\sigma(t)+\delta \hat{q}(t) \\
& =-\sigma(t)+G(\delta q(t)) \delta \tilde{q}(t)
\end{aligned}
$$

with its second time derivative

$$
\ddot{\sigma}(t)=-\dot{\sigma}(t)+\delta \tilde{q}_{0}(t) \delta \dot{q}(t)+E(\delta \dot{q}(t)) \delta \tilde{q}_{v}(t)+G(\delta q(t)) \delta \dot{\tilde{q}}(t)
$$

Once the proposed control law is implemented, the rate dynamics are obtained as

$$
\begin{aligned}
J \delta \dot{\omega}(t)= & -\delta \omega^{*}(t)\left(J \delta \omega(t)+\bar{J} \omega_{r}^{B}(t)\right)+\tilde{\eta}(t)+d(t) \\
& -\mathfrak{a}\left[\delta \tilde{q}_{0}(t) \delta q_{v}(t)+F(\delta q(t)) \delta \tilde{q}_{v}(t)\right]+\frac{\mathfrak{b}}{2} E^{T}(\dot{\sigma}(t)) G(\delta q(t)) \delta \tilde{q}(t),
\end{aligned}
$$

where $\tilde{\eta}=\hat{\eta}-\eta$. With the uncertainties, we now analyze

$$
V(t)=V_{1}(t)+\frac{c_{3}}{J_{m} \mathfrak{b}} V_{0}^{2}(t)
$$

where

$$
V_{1}(t)=c_{1} V_{0}(t)+\delta q_{v}^{T}(t) J \delta \omega(t)-2 c_{2} \delta \dot{q}^{T}(t) \dot{\sigma}(t)
$$

and, $c_{1} c_{2}$ and $c_{3}$ are positive constants to be determined. 
First, by differentiating Eq. (30), we have

$$
\begin{aligned}
\dot{V}_{0}= & \delta \omega^{T}\left[\mathfrak{a}\left(1-\delta \tilde{q}_{0}\right) \delta q_{v}-\mathfrak{a} F(\delta q) \delta \tilde{q}_{v}+\frac{\mathfrak{b}}{2} E^{T}(\dot{\sigma}) G(\delta q) \delta \tilde{q}+\tilde{\eta}+d\right] \\
& +\mathfrak{b} \dot{\sigma}^{T}\left[-\dot{\sigma}+\frac{\delta \tilde{q}_{0}}{2} E(\delta q) \delta \omega+E(\delta \dot{q}) \delta \tilde{q}_{v}+G(\delta q) \delta \dot{\tilde{q}}\right] \\
= & \delta \omega^{T}\left[\mathfrak{a}\left(1-\delta \tilde{q}_{0}\right) \delta q_{v}-\mathfrak{a} F(\delta q) \delta \tilde{q}_{v}+\tilde{\eta}+d\right] \\
& +\mathfrak{b} \dot{\sigma}^{T}[-\dot{\sigma}+G(\delta q) \delta \dot{\tilde{q}}]+\frac{\mathfrak{b}}{2} \delta \omega^{T}\left[E^{T}(\dot{\sigma}) E(\delta q)-E^{T}(\delta q) E(\dot{\sigma})\right] \delta \tilde{q}_{v}
\end{aligned}
$$

After arranging terms and applying the known or derived upper bounds for the measurement errors, external disturbances and parameter uncertainties, we obtain

$$
\dot{V}_{0} \leq-\mathfrak{b}\|\dot{\sigma}\|_{2}^{2}+\mathfrak{a}\left(1-\mu_{0}\right)\left\|\delta q_{v}\right\|_{2}\|\delta \omega\|_{2}+\mathfrak{b} \mu_{v}\|\delta \omega\|_{2}\|\dot{\sigma}\|_{2}+\left(\mathfrak{a} \mu_{v}+\mu_{\eta}+\mu_{d}\right)\|\delta \omega\|_{2}+\mathfrak{b} \mu_{\omega}\|\dot{\sigma}\|_{2}
$$

where

$$
\mu_{\eta}=4 \sqrt{J_{M} J_{\Delta}} v_{\omega}^{2} \mu_{v}^{2}+2 J_{M}\left(2 v_{\omega}^{2}+v_{\alpha}\right) \mu_{v}+\left(v_{\omega}^{2}+v_{\alpha}\right) \mu_{J}
$$

with $J_{\Delta}=J_{M}-J_{m}$, and $\mu_{J}$ such that $\|\hat{J}-J\|_{2} \leq \mu_{J}$. Note the following inequality:

$$
\begin{aligned}
\|C(\delta \hat{q})-C(\delta q)\|_{2} & =\left\|\left(C(\delta \tilde{q})-I_{3}\right) C(\delta q)\right\|_{2} \\
& =\left\|-2 F^{T}(\delta \tilde{q}) \delta \tilde{q}_{v}^{*} C(\delta q)\right\|_{2} \\
& \leq 2\left\|\delta \tilde{q}_{v}\right\|_{2}
\end{aligned}
$$

is used to obtain

$$
\|\tilde{\eta}\|_{2} \leq \mu_{\eta}
$$

In order to have in the right hand side of Eq. (39) a negative definite quadratic function of $z(t)$, we consider $V_{1}(t)$. The cross terms in $V_{1}(t)$ allow us to have non-positive quadratic terms in $\delta q_{v}(t)$ and $\delta \omega(t)$ within $\dot{V}_{1}(t)$. Since the upper bound of $\dot{V}_{1}(t)$ does not include a non-negative term in $(1-\delta q)$, one cannot say $V_{0}(t)$ is strictified. Instead, the term "partial" is introduced to describe this procedure. 
To ease the calculation, let us introduce

$$
\begin{aligned}
& N_{1}(t)=\delta q_{v}^{T}(t) J \delta \omega(t) \\
& N_{2}(t)=-\delta \dot{q}^{T}(t) \dot{\sigma}(t)
\end{aligned}
$$

whose time-derivatives are computed as

$$
\begin{aligned}
\dot{N}_{1}= & \frac{1}{2} \delta \omega^{T} F(\delta q) J \delta \omega-\mathfrak{a} \delta \tilde{q}_{0}\left\|\delta q_{v}\right\|_{2}^{2}+\frac{\mathfrak{b}}{2} \delta q_{v}^{T} E^{T}(\dot{\sigma}) \delta \hat{q} \\
& -\mathfrak{a} \delta q_{v}^{T} F(\delta q) \delta \tilde{q}_{v}+\delta q_{v}^{T}(\tilde{\eta}+d)-\delta q_{v}^{T} \delta \omega^{*} \bar{J} \omega_{r}^{B} \\
\dot{N}_{2}= & \delta \dot{q}^{T} \dot{\sigma}-\delta \tilde{q}_{0}\|\delta \dot{q}\|_{2}^{2}-\delta \dot{q}^{T} G(\delta q) \delta \dot{\tilde{q}}+\frac{1}{4}\|\delta \omega\|_{2}^{2} \dot{\sigma}^{T} \delta q-\frac{1}{2} \dot{\sigma}^{T} E(\delta q) \delta \dot{\omega} .
\end{aligned}
$$

Since $\|\delta q(t)\|_{2}=\|E(\delta q(t))\|_{2}=1$ and $\|F(\delta q(t))\|_{2} \leq 1$ for all $t \geq 0$, the upper bounds of Eqs. (45) and (46) are obtained as

$$
\begin{aligned}
\dot{N}_{1} \leq & -\mathfrak{a} \mu_{0}\left\|\delta q_{v}\right\|_{2}^{2}+\frac{J_{M}}{2}\|\delta \omega\|_{2}^{2}+\bar{J}_{M} v_{\omega}\left\|\delta q_{v}\right\|_{2}\|\delta \omega\|_{2}+\frac{\mathfrak{b}}{2}\left\|\delta q_{v}\right\|_{2}\|\dot{\sigma}\|_{2} \\
& +\left(\mathfrak{a} \mu_{v}+\mu_{\eta}+\mu_{d}\right)\left\|\delta q_{v}\right\|_{2}, \\
\dot{N}_{2} \leq & -\frac{\mu_{0}}{4}\|\delta \omega\|_{2}^{2}+\frac{\mathfrak{b}}{4 J_{m}}\|\dot{\sigma}\|_{2}^{2}+\frac{\mathfrak{a}}{2 J_{m}}\left\|\delta q_{v}\right\|_{2}\|\dot{\sigma}\|_{2}+\left(\frac{1}{2}+\frac{\bar{J}_{M} v_{\omega}}{2 J_{m}}\right)\|\delta \omega\|_{2}\|\dot{\sigma}\|_{2} \\
& +\left(\frac{1}{4}+\frac{\sqrt{J_{M} J_{\Delta}}}{2 J_{m}}\right)\|\delta \omega\|_{2}^{2}\|\dot{\sigma}\|_{2}+\frac{\mu_{\omega}}{2}\|\delta \omega\|_{2}+\frac{\left(\mathfrak{a} \mu_{v}+\mu_{\eta}+\mu_{d}\right)}{2 J_{m}}\|\dot{\sigma}\|_{2},
\end{aligned}
$$

Then, using Eq. (47) and (48), we have

$$
\begin{aligned}
\dot{V}_{1} \leq & -z^{\top} Q_{1} z+\gamma_{1}\left\|\delta q_{v}\right\|_{2}+\gamma_{2}\|\delta \omega\|_{2}+\gamma_{3}\|\dot{\sigma}\|_{2} \\
& +\left(\frac{1}{2}+\frac{\sqrt{J_{M} J_{\Delta}}}{J_{m}}\right) c_{2}\|\delta \omega\|_{2}^{2}\|\dot{\sigma}\|_{2}-\frac{1}{2}\left(c_{2} \mu_{0}-J_{M}\right)\left(1-\xi_{1}\right)\|\delta \omega\|_{2}^{2},
\end{aligned}
$$


where

$$
\begin{gathered}
\gamma_{1}=\mathfrak{a} \mu_{v}+\mu_{\eta}+\mu_{d}, \\
\gamma_{2}=c_{1} \gamma_{1}+c_{2} \mu_{\omega}, \\
\gamma_{3}=c_{1} \mathfrak{b} \mu_{\omega}+\frac{c_{2} \gamma_{1}}{J_{m}}
\end{gathered}
$$

and

$$
Q_{1}=\left[\begin{array}{ccc}
\mathfrak{a} \mu_{0} & -\frac{1}{2}\left(c_{1} \mathfrak{a}\left(1-\mu_{0}\right)+\bar{J}_{M} v_{\omega}\right) & -\frac{1}{2}\left(\frac{\mathfrak{b}}{2}+\frac{c_{2} \mathfrak{a}}{J_{m}}\right) \\
-\frac{1}{2}\left(c_{1} \mathfrak{a}\left(1-\mu_{0}\right)+\bar{J}_{M} v_{\omega}\right) & \frac{1}{2}\left(c_{2} \mu_{0}-J_{M}\right) \xi_{1} & -\frac{1}{2}\left(c_{1} \mathfrak{b} \mu_{v}+c_{2}+\frac{c_{2} \bar{J}_{M} v_{\omega}}{J_{m}}\right) \\
-\frac{1}{2}\left(\frac{\mathfrak{b}}{2}+\frac{c_{2} \mathfrak{a}}{J_{m}}\right) & -\frac{1}{2}\left(c_{1} \mathfrak{b} \mu_{v}+c_{2}+\frac{c_{2} \bar{J}_{M} v_{\omega}}{J_{m}}\right) & \left(c_{1}-\frac{c_{2}}{2 J_{m}}\right) \mathfrak{b}
\end{array}\right]
$$

with $\xi_{1} \in(0,1)$.

Remark 1. For the ideal set-point regulation problem with $v_{\omega}=v_{\alpha}=0$, the explicit inequalities

$$
c_{2}>J_{M}
$$

and

$$
c_{1}>\max \left\{\frac{c_{2}}{2 J_{m}}+\frac{1}{4 \mathfrak{a} \mathfrak{b}}\left(\frac{\mathfrak{b}}{2}+\frac{c_{2} \mathfrak{a}}{J_{m}}\right)^{2}+\frac{c_{2}^{2}}{2\left(c_{2}-J_{M}\right) \mathfrak{b} \xi_{1}}, \sqrt{\frac{c_{2}^{2}}{J_{m} \mathfrak{b}}+\frac{J_{M}}{2 \mathfrak{a}}}\right\}
$$

guarantee $V_{1}$ and $Q_{1}$ positive definite in the absence of uncertainties. Thus, the above conditions help to select proper values of $c_{1}$ and $c_{2}$ initially. Once reference tracking and uncertainties are considered, those lower bounds would be increased.

Next we analyze $V(t)$ defined in Eq. (36) as the final step. We assume that the positive constants $c_{1}$ and $c_{2}$ in $V_{1}$ are chosen such that $Q_{1}$ is positive definite and $V_{1}$ is nonnegative. Let

$$
N_{3}\left(\|\dot{\sigma}\|_{2}\right)=c_{3}\|\dot{\sigma}\|_{2}^{2}-\left(\frac{1}{2}+\frac{\sqrt{J_{M} J_{\Delta}}}{J_{m}}\right) c_{2}\|\dot{\sigma}\|_{2}+\frac{1}{2}\left(c_{2} \mu_{0}-J_{M}\right)\left(1-\xi_{1}\right)
$$


Then, using the inequalities

$$
\begin{aligned}
V_{0}(t) & \geq \frac{J_{m}}{2}\|\delta \omega(t)\|_{2}^{2} \\
\|\sigma(t)\|_{2} & \leq 1 \\
\|\dot{\sigma}(t)\|_{2} & \leq 2,
\end{aligned}
$$

together with Eq. (39), it is deduced that

$$
\dot{V} \leq-z^{T} Q_{1} z+\gamma_{1}\left\|\delta q_{v}\right\|_{2}+\left(\gamma_{2}+\gamma_{4} V_{0}\right)\|\delta \omega\|_{2}+\left(\gamma_{3}+\gamma_{5} V_{0}\right)\|\dot{\sigma}\|_{2}-\|\delta \omega\|_{2}^{2} N_{3}\left(\|\dot{\sigma}\|_{2}\right)
$$

where

$$
\begin{aligned}
\gamma_{4} & =\frac{2 c_{3}}{J_{m} \mathfrak{b}}\left[\mathfrak{a}\left(1-\mu_{0}\right)+\mathfrak{b} \mu_{v}+\gamma_{1}\right], \\
\gamma_{5} & =\frac{2 c_{3} \mu_{\omega}}{J_{m}}
\end{aligned}
$$

Moreover, since the choice

$$
c_{3}=\frac{c_{2}^{2}}{2\left(c_{2} \mu_{0}-J_{M}\right)\left(1-\xi_{1}\right)}\left(\frac{1}{2}+\frac{\sqrt{J_{M} J_{\Delta}}}{J_{m}}\right)^{2}
$$

leads to $N_{3}\left(\|\dot{\sigma}\|_{2}\right) \geq 0$ for any $\|\dot{\sigma}\|_{2} \in[0,2]$, we finally have

$$
\dot{V} \leq-z^{T} Q_{1} z+\gamma_{1}\left\|\delta q_{v}\right\|_{2}+\left(\gamma_{2}+\gamma_{4} V_{0}\right)\|\delta \omega\|_{2}+\left(\gamma_{3}+\gamma_{5} V_{0}\right)\|\dot{\sigma}\|_{2}
$$

In order to handle the nonnegative terms in Eq. (64), let us rewrite the inequality as

$\dot{V} \leq-z^{T} Q_{2} z-k_{1}\left\|\delta q_{v}\right\|_{2}^{2}-k_{2}\|\delta \omega\|_{2}^{2}-k_{3}\|\dot{\sigma}\|_{2}^{2}+\gamma_{1}\left\|\delta q_{v}\right\|_{2}+\left(\gamma_{2}+\gamma_{4} V_{0}\right)\|\delta \omega\|_{2}+\left(\gamma_{3}+\gamma_{5} V_{0}\right)\|\dot{\sigma}\|_{2}$, 
where

$$
\begin{aligned}
& k_{1}=\mathfrak{a} \mu_{0}\left(1-\xi_{2}\right), \\
& k_{2}=\frac{\left(c_{2} \mu_{0}-J_{M}\right)}{2} \xi_{1}\left(1-\xi_{3}\right), \\
& k_{3}=\left(c_{1}-\frac{c_{2}}{2 J_{m}}\right) \mathfrak{b}\left(1-\xi_{4}\right)
\end{aligned}
$$

with $\xi_{2}, \xi_{3}, \xi_{4} \in(0,1)$ and $Q_{2}=Q_{1}-\operatorname{diag}\left(k_{1}, k_{2}, k_{3}\right)$. For a vector $v \in \mathbb{R}^{n}, \operatorname{diag}(v) \in \mathbb{R}^{n \times n} \operatorname{denotes}$ a square diagonal matrix with the elements of $v$ on the main diagonal. Since the inequality

$$
\pm x^{T} P y \leq c x^{T} P x+\frac{1}{4 c} y^{T} P y
$$

holds for any $P=P^{T} \in \mathbb{R}^{n \times n}>0, x, y \in \mathbb{R}^{n}$ and $c>0$, we further obtain

$$
\dot{V} \leq-z^{T} Q_{2} z+\left(\frac{\gamma_{4}^{2}}{4 k_{2}}+\frac{\gamma_{5}^{2}}{4 k_{3}}\right) V_{0}^{2}+\left(\frac{\gamma_{2} \gamma_{4}}{2 k_{2}}+\frac{\gamma_{3} \gamma_{5}}{2 k_{3}}\right) V_{0}+\frac{1}{4}\left(\frac{\gamma_{1}^{2}}{k_{1}}+\frac{\gamma_{2}^{2}}{k_{2}}+\frac{\gamma_{3}^{2}}{k_{3}}\right) .
$$

Let us assume that there exist $c_{1}$ and $c_{2}$ such that $Q_{2}$ is positive definite. Then, by Lemma 3 in [25], there exists a positive definite matrix $\bar{Q}_{2} \in \mathbb{R}^{2 \times 2}$ such that

$$
z^{T}(t) Q_{2} z(t) \geq \bar{z}^{T}(t) \bar{Q}_{2} \bar{z}(t)
$$

Also, by Eq. (71), Lemma 4 in [25] and the fact that

$$
V_{0}(t) \leq 4 \mathfrak{a}+\frac{J_{M}}{2}\|\delta \omega(t)\|_{2}^{2}+\frac{\mathfrak{b}}{2}\|\dot{\sigma}(t)\|_{2}^{2},
$$

one can show that there exists a positive constant $k_{4}$ such that

$$
\dot{V} \leq-\frac{1}{k_{4}} V_{0}+\left(\frac{\gamma_{4}^{2}}{4 k_{2}}+\frac{\gamma_{5}^{2}}{4 k_{3}}\right) V_{0}^{2}+\left(\frac{\gamma_{2} \gamma_{4}}{2 k_{2}}+\frac{\gamma_{3} \gamma_{5}}{2 k_{3}}\right) V_{0}+\left[\frac{1}{4}\left(\frac{\gamma_{1}^{2}}{k_{1}}+\frac{\gamma_{2}^{2}}{k_{2}}+\frac{\gamma_{3}^{2}}{k_{3}}\right)+\frac{4 \mathfrak{a}}{k_{4}}\right]
$$


Once a parameter $\xi_{5} \in(0,1)$ is introduced, we obtain

$$
\dot{V}(t) \leq-\frac{\xi_{5}}{k_{4}} V_{0}(t)+N_{4}\left(V_{0}(t)\right)
$$

where

$$
N_{4}\left(V_{0}\right)=\varphi_{1} V_{0}^{2}-\varphi_{2} V_{0}+\varphi_{3}
$$

with

$$
\begin{aligned}
& \varphi_{1}=\frac{\gamma_{4}^{2}}{4 k_{2}}+\frac{\gamma_{5}^{2}}{4 k_{3}} \\
& \varphi_{2}=\frac{\left(1-\xi_{5}\right)}{k_{4}}-\left(\frac{\gamma_{2} \gamma_{4}}{2 k_{2}}+\frac{\gamma_{3} \gamma_{5}}{2 k_{3}}\right) \\
& \varphi_{3}=\frac{1}{4}\left(\frac{\gamma_{1}^{2}}{k_{1}}+\frac{\gamma_{2}^{2}}{k_{2}}+\frac{\gamma_{3}^{2}}{k_{3}}\right)+\frac{4 \mathfrak{a}}{k_{4}}
\end{aligned}
$$

To ensure the existence of $V_{0}>0$ such that $N_{4}\left(V_{0}\right) \leq 0$, let us assume the control gains $\mathfrak{a}$ and $\mathfrak{b}$ satisfy

$$
\varphi_{2}>2 \sqrt{\varphi_{1} \varphi_{3}}
$$

Let $r_{1}$ and $r_{2}$ be two positive distinct roots of the equation $N_{4}\left(V_{0}\right)=0$, and let $r_{1}<r_{2}$. Then, $r_{1} \leq V_{0}(t) \leq r_{2}$ implies

$$
\dot{V}(t) \leq-\frac{\xi_{5}}{k_{4}} V_{0}(t)
$$

From the inequality $V_{1}(t) \leq 2 c_{1} V_{0}(t)$, it is deduced that

$$
\frac{c_{3}}{J_{m} \mathfrak{b}} V_{0}^{2}(t) \leq V(t) \leq 2 c_{1} V_{0}(t)+\frac{c_{3}}{J_{m} \mathfrak{b}} V_{0}^{2}(t)
$$

After some algebra, we have

$$
\frac{\sqrt{\varphi_{5}^{2}+4 \varphi_{4} V(t)}-\varphi_{5}}{2 \varphi_{4}} \leq V_{0}(t) \leq \frac{\sqrt{4 \varphi_{4} V(t)}}{2 \varphi_{4}}
$$


where

$$
\begin{aligned}
\varphi_{4} & =\frac{c_{3}}{J_{m} \mathfrak{b}}, \\
\varphi_{5} & =2 c_{1} .
\end{aligned}
$$

Therefore, if

$$
\varphi_{4} r_{1}^{2}+\varphi_{5} r_{1} \leq V(t) \leq \varphi_{4} r_{2}^{2}
$$

then Eq. (80) follows. Again, for $V(t)$ to exist, a stricter condition for $\mathfrak{a}$ and $\mathfrak{b}$

$$
\varphi_{2} \geq \sqrt{\frac{\varphi_{1}^{4} \varphi_{5}^{2}}{\varphi_{2}^{2} \varphi_{4}^{2}} r_{1}^{2}+4 \varphi_{1} \varphi_{3}}
$$

is necessary. Now, we can conclude that the set $\left\{x \in \mathbb{Q} \times \mathbb{R}^{7} \mid V(x) \leq \varphi_{4} r_{2}^{2}\right\}$ is positively invariant and trajectories starting in this set will enter the residual set $\left\{x \in \mathbb{Q} \times \mathbb{R}^{7} \mid V(x) \leq \varphi_{4} r_{1}^{2}+\varphi_{5} r_{1}\right\}$ within a finite amount of time. Note that this result is only local because $\varphi_{4} r_{2}^{2}$ is finite [33].

Suppose $\delta q_{0}(t)$ is initially chosen such that $\delta q_{0}(0) \geq 0$. Then, at $t=0, V(0)$ is bounded by

$$
V(0) \leq c_{1} \bar{V}_{0}+J_{M}\left\|\delta q_{v}(0)\right\|_{2}\|\delta \omega(0)\|_{2}+\frac{c_{3}}{J_{m} \mathfrak{b}} \bar{V}_{0}^{2},
$$

where

$$
\bar{V}_{0}=2 \mathfrak{a}\left(1-\sqrt{1-\left\|\delta q_{v}(0)\right\|_{2}^{2}}\right)+\frac{J_{M}}{2}\|\delta \omega(0)\|_{2}^{2} .
$$

Hence, so long as $\left\|\delta q_{v}(0)\right\|_{2}$ and $\|\delta \omega(0)\|_{2}$ satisfy

$$
c_{1} \bar{V}_{0}+J_{M}\left\|\delta q_{v}(0)\right\|_{2}\|\delta \omega(0)\|_{2}+\frac{c_{3}}{J_{m} \mathfrak{b}} \bar{V}_{0}^{2} \leq \varphi_{4} r_{2}^{2},
$$

solutions are bounded for all $t \geq 0$.

In summary, the proposed controller

$$
u(t)=-\mathfrak{a} \delta \hat{q}_{v}(t)-\frac{\mathfrak{b}}{2} E^{T}(\delta \hat{q}(t)) \dot{\sigma}(t)+\left(C(\delta \hat{q}(t)) \omega_{r}(t)\right)^{*} \hat{J} C(\delta \hat{q}(t)) \omega_{r}(t)+\hat{J} C(\delta \hat{q}(t)) \dot{\omega}_{r}(t)
$$


regulates the tracking errors in the absence of uncertainties and makes them bounded in the presence of uncertainties if the control gains $\mathfrak{a}$ and $\mathfrak{b}$ are selected such that all the conditions described in this section are satisfied.

\section{Numerical Results}

We consider a CubeSat-class satellite whose dynamics are governed by Eq. (23) closed with the control law (32). It is assumed that the inertia matrix is given by [34]

$$
J=\left[\begin{array}{ccc}
0.0465 & -0.0007 & 0.0004 \\
-0.0007 & 0.0486 & -0.0021 \\
0.0004 & -0.0021 & 0.0482
\end{array}\right]
$$

and the measurement error bounds $\mu_{v}=\mu_{\omega}=0.01$ guarantee approximately 0.5 degree accuracy. Since existing nano star trackers have accuracy less than 0.03 degrees [34-36], this specification gives enough margin in the measurement errors. We also assume that the magnitude of external disturbance is bounded by $\mu_{d}=10^{-4}$ which is a few orders greater than the typical magnitude for satellites with similar size $[34,37]$. By letting $\left\|\delta q_{v}(0)\right\|_{2}=0.1,\|\delta \omega(0)\|_{2}=0.01$ and using Eq. (89), one can find a feasible gain region in which the control gains $\mathfrak{a}, \mathfrak{b}$ can be selected to ensure the boundedness of the closed-loop trajectories. In this section, we study both the stabilization and tracking problems. Since the stabilizing controller is independent of the inertia parameters, it is expected that the feasible gain region is not smaller than one obtained from general tracking cases.

First, consider the stabilization case by letting $q_{r}(t)=(1,0,0,0)$ and $\omega_{r}(t)=0$ which implies $v_{\omega}=v_{\alpha}=0$. To maximize the region, multiple values for each $\xi_{i}$ are considered. That is, the region in Fig. 2a is the union of the regions obtained using different $\xi$ values. Here, we restrict our gain range to a compact set $[0.01,0.3] \times[0.01,1.5] \subset \mathbb{R}^{2}$ for the computation. Note that it would be recommended to start with sufficiently bigger sets in order to search gain regions efficiently

To demonstrate the boundedness properties, we choose $(\mathfrak{a}, \mathfrak{b})=(0.2,0.8)$ and numerically integrate the closed-loop system using the following initial conditions: $q(0)=\hat{q}(0)=\sigma(0)=$ $(\sqrt{0.9925}, 0.05,0.05,0.05)$ and $\omega(0)=(-0.09,0.03,-0.03)$. The disturbance torque is assumed to 


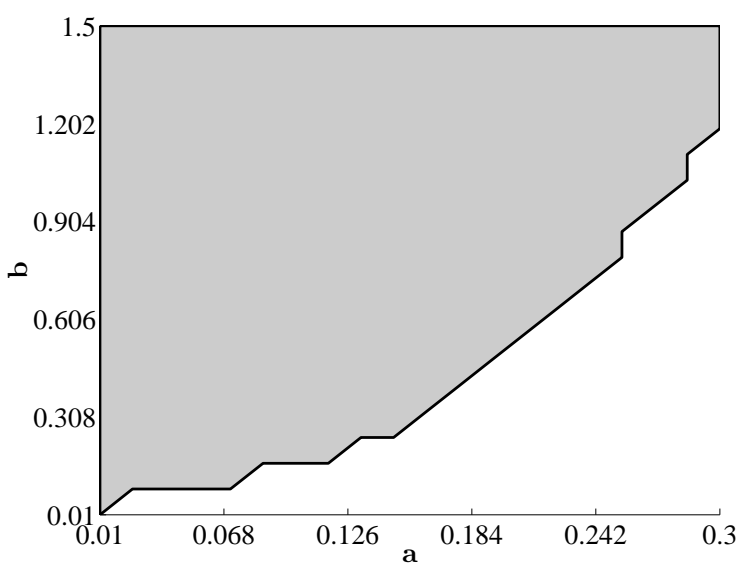

a) Stabilizing controller

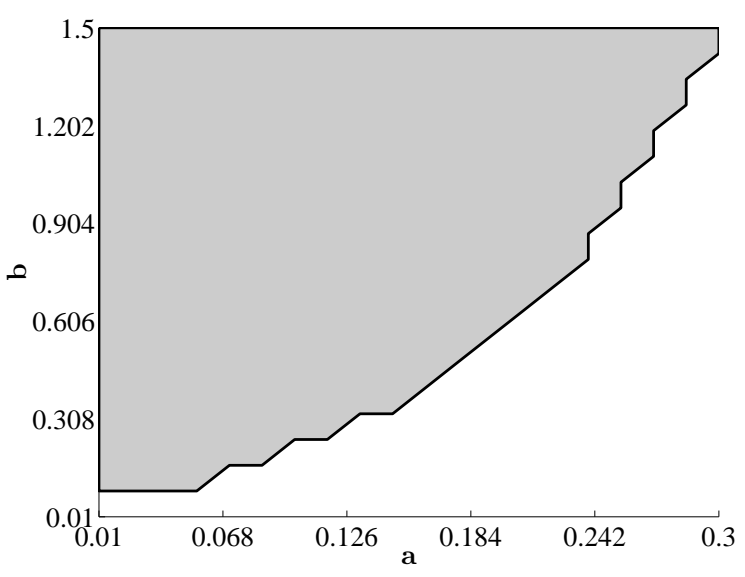

b) Tracking controller

Figure 2. Feasible Gain Regions for stabilizing and tracking controllers

be generated by gravity gradient, atmospheric drag and $J_{2}$ effect. The model is given by [20]

$$
d=\frac{3 \mu}{\|r\|_{2}^{5}} C(q) r^{*} J r-\frac{1}{2} \rho\|v\|_{2} C_{d} A r_{c}^{*} C(q) v+\frac{3 \mu J_{2} R_{e}^{2}}{2\|r\|_{2}^{7}} r_{c}^{*} C(q)\left[\begin{array}{c}
5 r_{x} r_{z}^{2}-3 r_{x}\|r\|_{2}^{2} \\
5 r_{y} r_{z}^{2}-3 r_{y}\|r\|_{2}^{2} \\
5 r_{z}^{3}-3 r_{z}\|r\|_{2}^{2}
\end{array}\right]
$$

where $r=\left(r_{x}, r_{y}, r_{z}\right) \in \mathbb{R}^{3}$ is the vector from the center of the Earth to the spacecraft represented in the inertial frame $\mathcal{F}_{I}, v \in \mathbb{R}^{3}$ is the spacecraft velocity vector in the inertial frame and $r_{c} \in \mathbb{R}^{3}$ is the vector from the spacecraft center of mass to the line of action of the force caused by the $J_{2}$ effect in the body-fixed frame $\mathcal{F}_{B}$. The other parameters in Eq. (92) are given by $\mu=398.6 \times 10^{12} \mathrm{~m}^{3} / \mathrm{s}^{2}$, $\rho=1.99 \times 10^{-14} \mathrm{~kg} / \mathrm{m}^{3}, C_{d}=2.5, A=0.04 \mathrm{~m}^{2}, R_{e}=6371.0 \times 10^{3} \mathrm{~m}$ and $J_{2}=1082.6 \times 10^{-6}$. The Earth-centered inertial frame $\mathcal{F}_{I}$ is defined such that the $\mathrm{x}$ axis points to the Sun and $\mathrm{z}$ axis is aligned with the axis of rotation of the Earth. The spacecraft is assumed to be in an circular orbit with the altitude $765 \mathrm{Km}$ and inclination $70^{\circ}$. For the numerical implementation, we choose $r_{c}=(0.01,0,0)$ so that the magnitude of the disturbance torque does not exceed the designed upper limit. As seen in Fig. 3a, the state norms do not converge to zero but are bounded. Fig. 3b illustrates the norm of the quaternion estimate error $\left\|\delta \tilde{q}_{v}(t)\right\|_{2}$ which is strictly less than $\mu_{v}$ during the simulation time. It is assumed that, at each simulation time $t$, the quaternion estimate error kinematics are driven by a 3-dimensional random vector $\tilde{\omega}$ with a uniform distribution of $\|\tilde{\omega}(t)\|_{2}$ 
over the closed interval $\left[0,2 v_{s}\right]$.

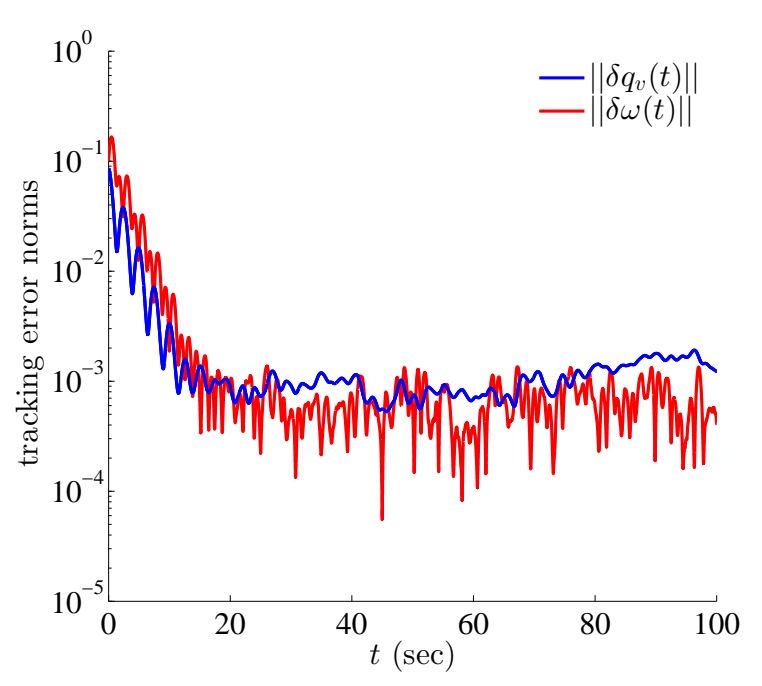

a) State norms

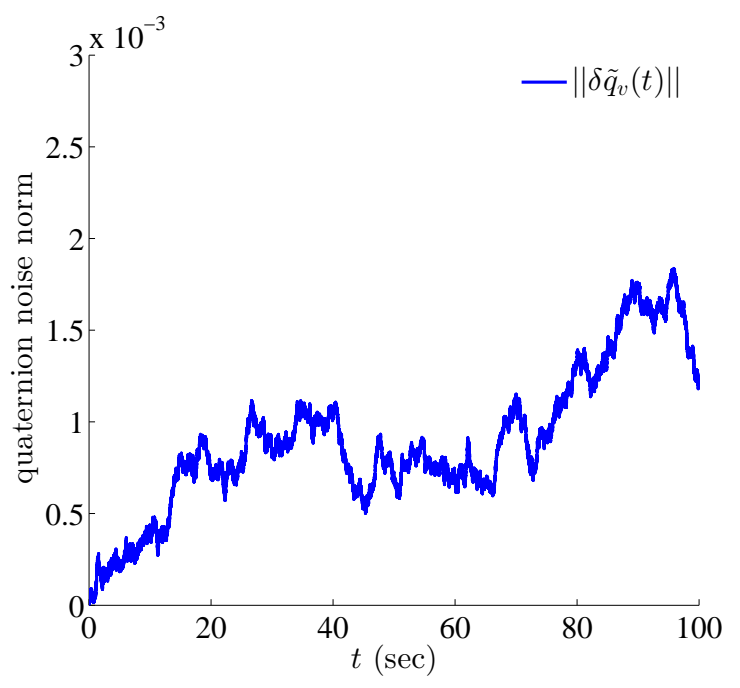

b) Quaternion noise norm

Figure 3. Time histories of $\left\|q_{v}(t)\right\|_{2},\|\omega(t)\|_{2}$ and $\left\|\delta \tilde{q}_{v}(t)\right\|_{2}$ for stabilization.

Next, we consider the tracking problem with the same uncertainty specifications. Suppose the reference angular velocity profile is given by $\omega_{r}(t)=0.05(\sin (0.5 t), \sin (0.5 t), \sin (0.5 t))$ with the initial reference quaternion $q_{r}(0)=(1,0,0,0)$. We obtain from the profile that $v_{\omega}=0.05 \sqrt{3}$ and $v_{\alpha}=0.5 v_{\omega}$. It is also assumed that the proposed tracking controller employs the estimated inertia matrix given by $J=\operatorname{diag}(0.046,0.049,0.048)$. Using the fact that $\mu_{J}=0.005$, a feasible gain region is computed as shown in Fig. 2b. We use the same gains and initial conditions described in the stabilization problem for the simulation. As illustrated in Fig. 4a, the tracking error norms are bounded.

\section{Conclusion}

This paper addresses the attitude tracking problem with the quaternion kinematics. It is assumed that only attitude vector measurements are available and are used to estimate the quaternion state. By employing the quaternion state and the output from a low-pass filter driven by the quaternion, a passivity-based tracking controller is proposed. Aided by the strictification technique, it is proved that one of the equilibrium points is asymptotically stable in the ideal case where there are no uncertainties. Even when measurement errors, and external disturbances introduce perturbations 


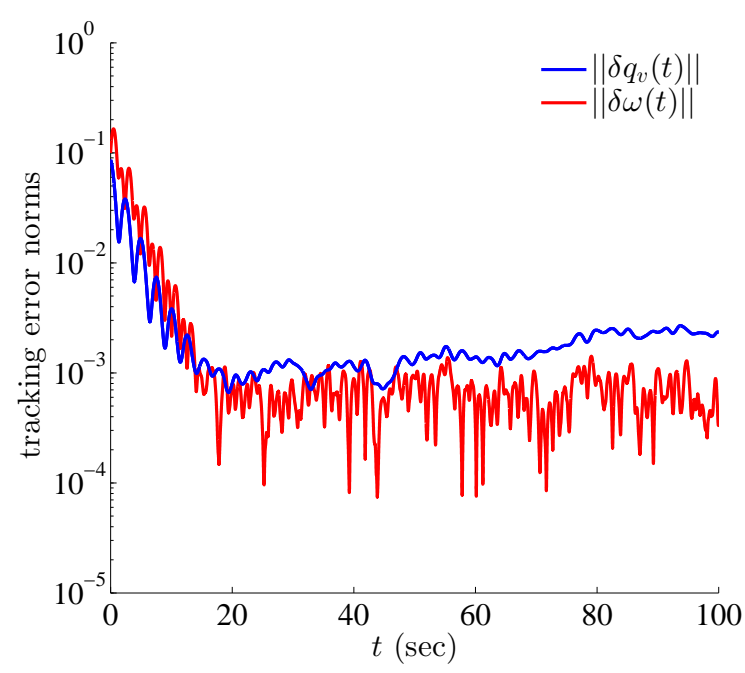

a) Tracking error norms

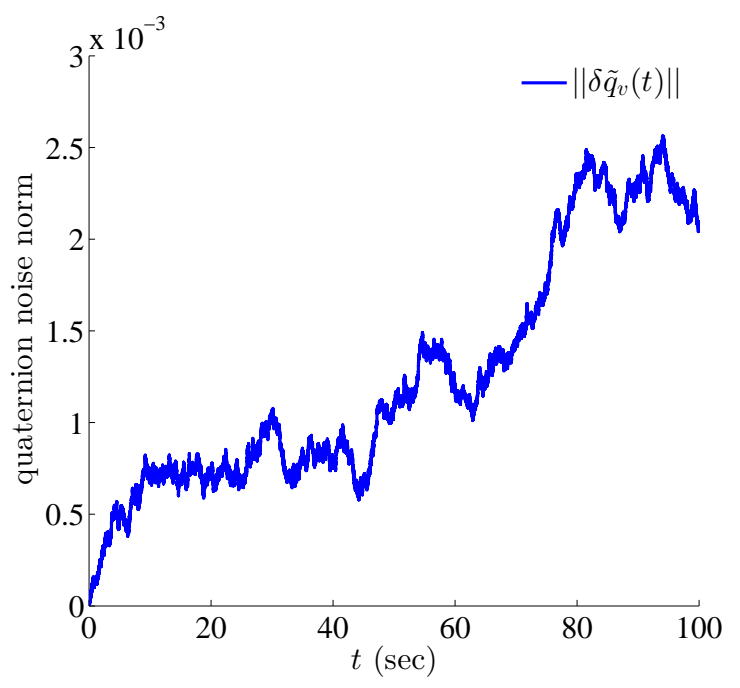

b) Quaternion noise norm

Figure 4. Time histories of $\left\|\delta q_{v}(t)\right\|_{2},\|\delta \omega(t)\|_{2}$ and $\left\|\delta \tilde{q}_{v}(t)\right\|_{2}$ for tracking.

along with model mismatch in the dynamics, a positive invariant set and a residual set are established in use of known bounds for the uncertainties. Then sufficient conditions for the initial states and the feedback gains that guarantee boundedness of the closed-loop trajectories through Lyapunov analysis are provided.

\section{References}

[1] Wen, J.-Y. and Kreutz-Delgado, K., “The attitude control problem,” Automatic Control, IEEE Transactions on, Vol. 36, No. 10, 1991, pp. 1148-1162.

doi:10.1109/9.90228

[2] Wallsgrove, R. J. and Akella, M. R., "Globally stabilizing saturated attitude control in the presence of bounded unknown disturbances," Journal of Guidance, Control, and Dynamics, Vol. 28, No. 5, 2005, pp. 957-963.

doi:10.2514/1.9980

[3] Wie, B., Space vehicle dynamics and control, AIAA, 2nd ed., 2008.

[4] Seo, D. and Akella, M. R., "High-performance spacecraft adaptive attitude-tracking control through attracting-manifold design," Journal of Guidance, Control, and Dynamics, Vol. 31, 
No. 4, 2008, pp. 884-891.

doi:10.2514/1.33308

[5] Mercker, T. H. and Akella, M. R., "Rigid-body attitude tracking with vector measurements and unknown gyro bias," Journal of guidance, control, and dynamics, Vol. 34, No. 5, 2011, pp. $1474-1484$.

doi:10.2514/1.53111

[6] Lizarralde, F. and Wen, J. T., "Attitude control without angular velocity measurement: A passivity approach," Automatic Control, IEEE Transactions on, Vol. 41, No. 3, 1996, pp. 468472.

doi:10.1109/9.486654

[7] Tsiotras, P., "Further passivity results for the attitude control problem," IEEE Transactions on Automatic Control, Vol. 43, No. 11, 1998, pp. 1597-1600.

doi: $10.1109 / 9.728877$

[8] Akella, M. R., "Rigid body attitude tracking without angular velocity feedback," Systems \& Control Letters, Vol. 42, No. 4, 2001, pp. 321-326.

doi:10.1016/S0167-6911(00)00102-X

[9] Subbarao, K. and Akella, M. R., "Differentiator-free nonlinear proportional-integral controllers for rigid-body attitude stabilization," Journal of guidance, control, and dynamics, Vol. 27, No. 6, 2004, pp. 1092-1096.

doi:10.2514/1.8366

[10] Akella, M. R., Valdivia, A., and Kotamraju, G. R., "Velocity-free attitude controllers subject to actuator magnitude and rate saturations," Journal of Guidance, Control, and Dynamics, Vol. 28, No. 4, 2005, pp. 659-666.

doi:10.2514/1.8784

[11] Bin, X., Mu, H., Dong, L., Cuijie, C., and Kaiyan, Y., "Output feedback attitude tracking control for a rigid spacecraft with dynamic uncertainty," Control Conference, 2008. CCC 
2008. 27th Chinese, IEEE, 2008, pp. 464-468.

doi:10.1109/CHICC.2008.4605788

[12] Tayebi, A., "Unit quaternion-based output feedback for the attitude tracking problem," Automatic Control, IEEE Transactions on, Vol. 53, No. 6, 2008, pp. 1516-1520. doi:10.1109/TAC.2008.927789

[13] Costic, B. T., Dawson, D. M., De Queiroz, M. S. and Kapila, V., "Quaternion-based adaptive attitude tracking controller without velocity measurements," Journal of Guidance, Control, and Dynamics, Vol. 24, No. 6, 2001, pp. 1214-1222.

doi:10.2514/2.4837

[14] Crassidis, J. L., Markley, F. L., and Cheng, Y., "Survey of nonlinear attitude estimation methods," Journal of Guidance, Control, and Dynamics, Vol. 30, No. 1, 2007, pp. 12-28. doi:10.2514/1.22452

[15] Lerner, G. M., “Three-axis attitude determination," Spacecraft Attitude Determination and Control, Vol. 73, 1978, pp. 420-428.

[16] Shuster, M. D. and Oh, S., "Three-axis attitude determination from vector observations," Journal of Guidance, Control, and Dynamics, Vol. 4, No. 1, 1981, pp. 70-77. doi:10.2514/3.19717

[17] Markley, F. L., "Attitude determination using vector observations: A fast optimal matrix algorithm," Journal of the Astronautical Science, Vol. 41, No. 2, 1993, pp. 261-280.

[18] Tayebi, A., Roberts, A., and Benallegue, A., "Inertial vector measurements based velocityfree attitude stabilization," Automatic Control, IEEE Transactions on, Vol. 58, No. 11, 2013, pp. 2893-2898.

doi:10.1109/TAC.2013.2256689 
[19] Thakur, D. and Akella, M. R., “Gyro-Free Rigid-Body Attitude Stabilization Using only Vector Measurements," Journal of Guidance, Control, and Dynamics, 2014, pp. 1-8. doi:10.2514/1.G000623

[20] Schlanbusch, R., Loria, A., Kristiansen, R., and Nicklasson, P. J., "PD+ attitude control of rigid bodies with improved performance," Decision and Control (CDC), 2010 49th IEEE Conference on, IEEE, 2010, pp. 7069-7074.

doi:10.1109/CDC.2010.5717227

[21] Schlanbusch, R., Loria, A., Kristiansen, R., and Nicklasson, P. J., "PD+ based output feedback attitude control of rigid bodies," Automatic Control, IEEE Transactions on, Vol. 57, No. 8, 2012, pp. 2146-2152.

doi:10.1109/TAC.2012.2183189

[22] Chaillet, A. and Loría, A., "Uniform semiglobal practical asymptotic stability for nonautonomous cascaded systems and applications," Automatica, Vol. 44, No. 2, 2008, pp. 337347.

doi:10.1016/j.automatica.2007.05.019

[23] Xian, B., Diao, C., Zhao, B. and Zhang, Y., "Nonlinear robust output feedback tracking control of a quadrotor UAV using quaternion representation," Nonlinear Dynamics, Vol. 79, No. 4, 2015, pp. 2735-2752.

doi:10.1007/s11071-014-1843-X

[24] Bandyopadhyay, S., Chung, S. and Hadaegh, F. Y., "Nonlinear attitude control of spacecraft with a large captured object," Journal of Guidance, Control, and Dynamics, Vol. 39, No. 4, 2016, pp. 754-769.

doi:10.2514/1.G001341

[25] Mazenc, F., Yang, S., and Akella, M. R., "Output feedback, attitude dynamics, robustness," Control Conference (ECC), 2015 European, IEEE, 2015, pp. 1249-1254.

doi:10.1109/ECC.2015.7330711 
[26] Yang, S., Mazenc, F., and Akella, M. R., "Velocity-Free Attitude Stabilization with Measurement Errors," AAS/AIAA Astrodynamics Specialist Conference, American Astronautical Soc. Paper 2015-685, 2015.

[27] Malisoff, M. and Mazenc, F., Constructions of Strict Lyapunov functions, Communications and Control Engineering Series, Springer, New York, 2009.

[28] Mazenc, F. and Nesic, D., "Lyapunov functions for time-varying systems satisfying generalized conditions of Matrosov theorem," Mathematics of Control, Signals, and Systems, Vol. 19, No. 2, 2007, pp. 151-182.

doi:10.1007/s00498-007-0015-7

[29] Mazenc, F. and Akella, M. R., "Quaternion-based stabilization of attitude dynamics subject to pointwise delay in the input," American Control Conference (ACC), 2014, IEEE, 2014, pp. 4877-4882.

doi:10.1109/ACC.2014.6858703

[30] Schaub, H. and Junkins, J. L., Analytical Mechanics of Space Systems, AIAA Education Series, AIAA, Reston, VA, 2003, Chap. 3.

[31] Seo, D. and Akella, M. R., "Separation property for the rigid-body attitude tracking control problem," Journal of Guidance, Control, and Dynamics, Vol. 30, No. 6, 2007, pp. 15691576.

doi:10.2514/1.30296

[32] Mortari, D. and Akella, M. R., "Discrete and Continuous Time Adaptive Angular Velocity Estimators," 25th AAS/AIAA Space Flight Mechanics Meeting, American Astronautical Soc. Paper 2015-254, 2015.

[33] Khalil, H. K. and Grizzle, J., Nonlinear systems, Prentice hall New Jersey, 3rd ed., 2002.

[34] Johnston-Lemke, B., High Performance Attitude Determination and Control for Nanosatellites Missions, Ph.D. thesis, University of Toronto, 2011. 
[35] Erlank, A. O., Development of CubeStar A CubeSat-Compatible Star Tracker, Ph.D. thesis, Stellenbosch University, 2013.

[36] McBryde, C. R. and Lightsey, E. G., "A star tracker design for CubeSats," Aerospace Conference, 2012 IEEE, IEEE, 2012, pp. 1-14.

doi:10.1109/AERO.2012.6187242

[37] Inamori, T., Sako, N., and Nakasuka, S., "Magnetic dipole moment estimation and compensation for an accurate attitude control in nano-satellite missions," Acta Astronautica, Vol. 68, No. 11, 2011, pp. 2038-2046.

doi:10.1016/j.actaastro.2010.10.022 